PROCEEDINGS OF THE

AMERICAN MATHEMATICAL SOCIETY

Volume 138, Number 8, August 2010, Pages 2889-2895

S 0002-9939(10)10385-2

Article electronically published on March 29, 2010

\title{
A GENERALIZATION OF CARTAN'S THEOREM ON ISOPARAMETRIC CUBICS
}

\author{
VLADIMIR G. TKACHEV \\ (Communicated by Chuu-Lian Terng)
}

\begin{abstract}
We generalize the well-known result of É. Cartan on isoparametric cubics by showing that a homogeneous cubic polynomial solution of the eiconal equation $|\nabla f|^{2}=9|x|^{4}$ must be rotationally equivalent to either $x_{n}^{3}-3 x_{n}\left(x_{1}^{2}+\ldots+x_{n-1}^{2}\right)$ or to one of four exceptional Cartan cubic polynomials in dimensions $n=5,8,14,26$.
\end{abstract}

\section{INTRODUCTION}

In his paper [1] É. Cartan found all cubic homogeneous polynomials in $\mathbb{R}^{n}, n \geq 3$, satisfying the isoparametric equations

$$
\begin{gathered}
|\nabla f(x)|^{2}=9|x|^{4}, \\
\Delta f(x)=0 .
\end{gathered}
$$

Amazingly, the cubic solutions of (1.1)-(1.2) can be described by means of four real division algebras $\mathbb{F}_{d}$ of dimension $d$, where $\mathbb{F}_{1}=\mathbb{R}$ (reals), $\mathbb{F}_{2}=\mathbb{C}$ (complexes), $\mathbb{F}_{4}=\mathbb{H}$ (quaternions) and $\mathbb{F}_{8}=\mathbb{O}$ (octonians). Cartan proved that for a cubic solution to exist the dimension $n$ must be $5,8,14$ or 26 , i.e.

$$
n=3 d+2, \quad d=1,2,4,8,
$$

and, in this case, the solution is congruent (i.e. rotationally equivalent) to one of the following polynomials (cf. [1, p. 34]):

$$
\begin{aligned}
f_{d}(x) & =x_{n}^{3}-3 x_{n} x_{n-1}^{2}+\frac{3}{2} x_{n}\left(X_{0} \bar{X}_{0}+X_{1} \bar{X}_{1}-2 X_{2} \bar{X}_{2}\right) \\
& +\frac{3 \sqrt{3}}{2} x_{n-1}\left(X_{0} \bar{X}_{0}-X_{1} \bar{X}_{1}\right)+\frac{3 \sqrt{3}}{2}\left(\left(X_{0} X_{1}\right) X_{2}+\bar{X}_{2}\left(\bar{X}_{1} \bar{X}_{0}\right)\right),
\end{aligned}
$$

where $x=\left(X_{0}, X_{1}, X_{2}, x_{n-1}, x_{n}\right)$ and vector $X_{k}=\left(x_{k d+1}, \ldots, x_{k d+d}\right)$ are identified with the corresponding elements of $\mathbb{F}_{d}, k=0,1,2$, and $\bar{X}$ denotes the conjugate of $X$ in $\mathbb{F}_{d}$. It is not hard to prove also that all the Cartan polynomials are irreducible.

In dimension $n=2$ there is also a reducible polynomial satisfying (1.1)-(1.2),

$$
f_{0}(x)=x_{2}^{3}-3 x_{2} x_{1}^{2}=\operatorname{Re}\left(x_{2}+x_{1} \sqrt{-1}\right)^{3},
$$

Received by the editors August 20, 2009.

2010 Mathematics Subject Classification. Primary 53C42, 35F20, 17A35; Secondary 15A63, $17 \mathrm{~A} 75$.

Key words and phrases. Cartan's theorem, division algebras, composition formulas, quadratic maps, eiconal equation, minimal cubic cones.

(C)2010 American Mathematical Society 
which, though having no evident relation to the division algebras, can be thought of (at least formally) as the member of the above family corresponding to $d=0$, where all $X_{i}$ are supposed to be zero. It is easy to see that in higher dimensions $n \geq 3$, this polynomial $f_{0}(x)$ gives rise to a new family of (reducible) solutions of the eiconal equation (1.1) alone, namely

$$
f_{0}(x)=x_{n}^{3}-3 x_{n}\left(x_{1}^{2}+\cdots+x_{n-1}^{2}\right) .
$$

Note also that $f_{0}$ is not congruent to any of the $f_{d}$ in the corresponding dimensions because all $f_{d}$ are harmonic, while $\Delta f_{0}=6(2-n) x_{n} \neq 0$ for $n \geq 3$.

Our main result is the following characterization of cubic solutions of equation (1.1) alone.

Theorem 1.1. Any homogeneous cubic polynomial satisfying the eiconal equation $|\nabla f|^{2}=9|x|^{4}$ is rotationally equivalent to either $x_{n}^{3}-3 x_{n}\left(x_{1}^{2}+\ldots+x_{n-1}^{2}\right)$ or to one of the exceptional Cartan cubic polynomials $f_{d}(x)$ in dimensions $n=5,8,14,26$. In particular, irreducible cubic solutions of (1.1) can exist only in dimensions $n=$ $5,8,14$ and 26 .

Remark 1.2. It is well known that for $d \neq 0$ the focal varieties $f_{d}=0$ are minimal cones in $\mathbb{R}^{3 d+2}$ (i.e. immersed submanifolds having zero mean curvature). So far, these four Cartan cones are the only known examples of minimal cubics besides the cubic $2 x_{1} x_{2} x_{3}+\left(x_{1}^{2}-x_{2}^{2}\right) x_{4}$ (a member of Lawson's family of algebraic minimal surfaces in $\mathbb{R}^{4}$ given in [3] ) and two additional cubics, each in dimensions 9 and 15 , found by Wu-yi Hsiang in [2]. In a forthcoming paper [5] we provide a classification of minimal cubics in $\mathbb{R}^{n}$, and Theorem 1.1 above plays a crucial role in constructing the so-called exceptional family of minimal cubics in $\mathbb{R}^{3 k}$.

\section{Symmetric COMPOSITION FORMULAS}

Recall that a composition formula of size $[r, s, m]$ over the field of real numbers (see [4]) is an identity

$$
\sum_{k=1}^{m} b_{k}^{2}(x, y)=|x|^{2}|y|^{2}, \quad x \in \mathbb{R}^{r}, y \in \mathbb{R}^{s},
$$

where $b_{k}(x, y)$ are real bilinear forms and $|x|^{2}=\langle x, x\rangle$ is the usual Euclidean norm of $x$. It is well known that the existence of a composition formula of size $[r, s, m]$ is equivalent to solvability of the Hurwitz matrix equations

$$
\begin{gathered}
A_{i}^{\mathrm{t}} A_{i}=1_{s}, \quad 1 \leq i \leq r, \\
A_{i}^{\mathrm{t}} A_{j}+A_{j}^{\mathrm{t}} A_{i}=0, \quad i \neq j
\end{gathered}
$$

(see, for instance, [4). Here $A_{i} \in \mathbb{R}^{m \times s}$ is a matrix of size $m \times s$ with real entries, $A^{\mathrm{t}}$ denote the transpose matrix, and $1_{k}$ stands for the unit matrix in $\mathbb{R}^{k \times k}$. It follows from (2.1) that $m \geq \max \{r, s\}$.

If $\max \{r, s\}=m$, say $s=m$, then the celebrated Hurwitz-Radon theorem states that a composition formula of size $[r, m, m]$ exists (equivalently, the Hurwitz matrix system of size $[r, m, m]$ is solvable) iff

$$
r \leq \rho(m),
$$


where the Hurwitz-Radon function $\rho(m)$ is defined for positive integers $m \geq 1$ by the formula

$$
\rho(m)=8 a+2^{b}, \quad \text { where } m=2^{4 a+b} \cdot \text { odd }, 0 \leq b \leq 3,
$$

and $\rho(m)=0$ otherwise. In particular, for positive integers we always have $\rho(m) \leq$ $m$ with equality only if $m=1,2,4,8$. Another useful observation is that $\rho(m)=1$ if and only if $m$ is odd.

We shall need an analogue of the Hurwitz-Radon function for symmetric solutions of (2.1)-(2.2). Given $m \geq 1$ we define $\rho_{\text {symm }}(m)$ as the maximal possible $r$ such that the Hurwitz matrix equations are solvable for symmetric matrices $A_{i} \in \mathbb{R}^{m \times m}$, $i=1, \ldots, r$.

Proposition 2.1. For any $m \geq 1$,

$$
\rho_{\text {symm }}(m)=1+\rho\left(\frac{m}{2}\right) .
$$

Moreover, if $\left\{A_{i}\right\}_{1 \leq i \leq r}$ is a symmetric solution of (2.1) -(2.2) for $r=\rho_{\mathrm{symm}}(m) \geq$ 2 , then all the matrices are trace free: trace $A_{i}=0$.

Proof. First suppose that $\rho_{\text {symm }}(m)=1$. Then $m$ must be an odd number, because otherwise $m=2 k, k \in \mathbb{Z}$, and the two matrices

$$
A_{1}=\left(\begin{array}{cc}
-1_{k} & 0 \\
0 & 1_{k}
\end{array}\right), \quad A_{2}=\left(\begin{array}{cc}
0 & 1_{k} \\
1_{k} & 0
\end{array}\right)
$$

provide a symmetric solution of (2.1)-(2.2) with $r=2$. Thus $m$ is odd and it follows from (2.3) that $\rho(m)=1$. This proves (2.4) for $\rho_{\text {symm }}(m)=1$.

Now let us consider the case $r:=\rho_{\mathrm{symm}}(m) \geq 2$. Then we can find a symmetric solution $\left\{A_{i}\right\}_{1 \leq i \leq r}$ of (2.1)-(2.2). Without loss of generality we can assume that $A_{r}$ has the diagonal form, say $A_{r}=\operatorname{diag}\left(a_{1}, \ldots, a_{m}\right)$, where $a_{i} \in \mathbb{R}$. Then (2.1) implies that $a_{i}^{2}=1$; that is, after a suitable rotation we get

$$
A_{r}=1_{t} \oplus(-1)_{m-t}, \quad 0 \leq t \leq m
$$

We claim that $t(m-t) \neq 0$. Indeed, if $t=0$ or $t=m$, then $A_{r}= \pm 1_{m}$; hence applying (2.2) to $A_{i}$ and $A_{r}$ we find that $A_{i}^{\mathrm{t}}+A_{i}=0,1 \leq i \leq r-1$, which in its turn implies that $A_{i}=0$ because the $A_{i}$ are symmetric. But the latter contradicts (2.1) for $r \geq 2$. Hence $A_{r}$ has eigenvalues of both signs, i.e. $1 \leq t \leq m-1$ in (2.5).

Write the remaining $A_{i}$ in the block form associated with the polarization of $\mathbb{R}^{m}$ given by (2.5),

$$
A_{i}=\left(\begin{array}{cc}
C_{i} & E_{i} \\
E_{i}^{\mathrm{t}} & D_{i}
\end{array}\right), \quad 1 \leq i \leq r-1
$$

Here $C_{i} \in \mathbb{R}^{t \times t}$ and $D_{i} \in \mathbb{R}^{(m-t) \times(m-t)}$ are symmetric matrices, and $E_{i} \in \mathbb{R}^{t \times(m-t)}$. Applying again (2.2) to $A_{i}$ and $A_{r}$ we find immediately that $C_{i}$ and $D_{i}$ are zero matrices for $i \leq r-1$. Furthermore, (2.1) yields

$$
E_{i} E_{i}^{\mathrm{t}}=1_{t}, \quad E_{i}^{\mathrm{t}} E_{i}=1_{m-t},
$$

and setting $1 \leq i, j \leq r-1$ in (2.2) we get

$$
E_{i} E_{j}^{\mathrm{t}}+E_{j} E_{i}^{\mathrm{t}}=0 .
$$

Now observe that identity $E_{i} E_{i}^{\mathrm{t}}=1_{t}$ implies $m-t \geq t$, and similarly, $E_{i}^{\mathrm{t}} E_{i}=$ $1_{m-t}$ implies $t \geq m-t$. Hence $m=2 t$; in particular, $m$ is an even number. It follows that all $E_{i}$ are quadratic matrices and equations (2.6)-(2.7) are equivalent 
to the Hurwitz matrix equations of size $[r-1, t, m-t] \equiv\left[r-1, \frac{m}{2}, \frac{m}{2}\right]$. This implies by the definition of $\rho$ that $r-1 \leq \rho(m / 2)$, i.e.

$$
\rho_{\text {symm }}(m)-1 \leq \rho(m / 2) .
$$

In order to prove the inverse inequality, let us fix an even $m \geq 2$ and set

$$
r:=\rho(m / 2)+1 \geq 2 .
$$

Let $\left\{E_{i}\right\}_{1 \leq i \leq r}$ be an arbitrary solution of (2.1) -(2.2) of size $\left[r-1, \frac{m}{2}, \frac{m}{2}\right]$. Then it is easy to check that the symmetric matrices

$$
A_{i}=\left(\begin{array}{cc}
0 & E_{i} \\
E_{i}^{\mathrm{t}} & 0
\end{array}\right), \quad 1 \leq i \leq r-1, \quad A_{r}=1_{m / 2} \oplus(-1)_{m / 2}
$$

give a solution to (2.6)-(2.7) of size $[r, m, m]$. Thus $\rho_{\text {symm }}(m) \geq r=\rho(m / 2)+1$, which finishes the proof of (2.4).

The last statement of the proposition easily follows from the block form of $A_{i}$ and the fact that trace is invariant with respect to orthogonal transformations.

\section{Proof of Theorem 1.1}

Let $f$ be any cubic polynomial satisfying (1.1). Then $f \not \equiv 0$ and it can be brought into the normal form, i.e.

$$
f(x)=x_{n}^{3}+3 x_{n} A(\bar{x})+3 B(\bar{x}), \quad \bar{x}=\left(x_{1}, \ldots, x_{n-1}\right),
$$

where $A$ is a quadratic form and $B$ is a cubic form in $\bar{x}$. Indeed, the maximum value of $f(x)$ on the unit sphere $|x|=1$ is strictly positive and attained at some point $x^{0}$. Then $\nabla f\left(x^{0}\right)=c x^{0}$; hence by homogeneity of $f$,

$$
c=\left\langle x^{0}, \nabla f\left(x^{0}\right)\right\rangle=3 f\left(x^{0}\right) \neq 0,
$$

and it is easily shown that in the new orthogonal coordinates with $x_{0}$ being the $n$th vector, $f$ takes the form (3.1).

Equating $|\nabla f|^{2}$ to $9|x|^{2}$ yields

$$
x_{n}^{2}\left(2 A+|\nabla A|^{2}-2|\bar{x}|^{2}\right)+2 x_{n}\langle\nabla A, \nabla B\rangle+\left(A^{2}+|\nabla B|^{2}-|\bar{x}|^{4}\right)=0,
$$

where $\bar{x}=\left(x_{1}, \ldots, x_{n-1}\right)$. Thus

$$
\begin{gathered}
2 A+|\nabla A|^{2}-2|\bar{x}|^{2}=0, \\
\langle\nabla A, \nabla B\rangle=0, \\
A^{2}+|\nabla B|^{2}=|\bar{x}|^{4} .
\end{gathered}
$$

We can assume without loss of generality that $A$ is given in the diagonal form, say $A(\bar{x})=\operatorname{diag}\left(a_{1}, \ldots, a_{n-1}\right)$, so that (3.2) yields $2 a_{i}^{2}+a_{i}-1=0$. This implies that $a_{i}$ is either $\frac{1}{2}$ or -1 . We redenote the coordinates such that

$$
A(\bar{x})=\frac{1}{2} \sum_{i=1}^{p} \xi_{i}^{2}-\sum_{j=1}^{q} \eta_{i}^{2}, \quad p+q=n-1, \quad \bar{x}=(\xi, \eta) .
$$

Denote by $V_{1}$ and $V_{2}$ the corresponding eigenspaces of dimensions $p$ and $q$, respectively. The thus obtained polarization $V \equiv \mathbb{R}^{n-1}=V_{1} \oplus V_{2}$ induces the corresponding decompositions in the tensor products, in particular,

$$
V^{* \otimes 3} \simeq V^{3,0} \oplus V^{2,1} \oplus V^{1,2} \oplus V^{0,3}, \quad V^{i, j}=V_{1}^{* \otimes i} \otimes V_{2}^{* \otimes(3-i)} .
$$


According to the latter decomposition we have for the cubic form $B$,

$$
B=B_{3,0}+B_{2,1}+B_{1,2}+B_{0,3},
$$

where $B_{i, 3-i} \equiv B_{i, 3-i}(\xi, \eta) \in V^{i, 3-i}$ are linearly independent cubic forms. By homogeneity one finds

$$
\begin{aligned}
\left\langle\xi, \nabla B_{i, 3-i}\right\rangle & =i B_{i, 3-i}, \\
\left\langle\eta, \nabla B_{i, 3-i}\right\rangle & =(3-i) B_{i, 3-i}
\end{aligned}
$$

hence by virtue of (3.3),

$$
\langle\nabla A, \nabla B\rangle=\left\langle\xi-2 \eta, \sum_{i=0}^{3} \nabla B_{i, 3-i}\right\rangle=-6 B_{3,0}-3 B_{1,2}+3 B_{0,3}=0 .
$$

It follows from the linear independence of $B_{i, 3-i}$ that $B_{3,0}=B_{1,2}=B_{0,3}=0$. Thus $B \in V^{2,1}$, i.e.

$$
B \equiv B_{2,1}=\sum_{i=1}^{q} \eta_{i} Q_{i}(\xi)
$$

where $Q_{i}(\xi) \in V_{2}^{\otimes *^{2}}$ is a quadratic form in $\xi$.

Note that $q \geq 1$, since otherwise we would have $B \equiv 0$ and by virtue of (3.4), $A^{2}=|\bar{x}|^{4}$, which would imply a contradiction to (3.5), because $A=\frac{1}{2}|\bar{\xi}|^{2} \equiv \frac{1}{2}|\bar{x}|^{2}$ for $q=0$. Thus $q=\operatorname{dim} V_{2} \geq 1$.

Note also that if $\operatorname{dim} V_{1}=p=0$, then $B=0$ and $A=-|\eta|^{2}$. It is easy to check that (3.2)-(3.4) turn into identities and the corresponding $f$ becomes the solution of (1.1) in the form (1.3).

It only remains to treat the case when both $V_{1}$ and $V_{2}$ are nontrivial: $\operatorname{dim} V_{k} \geq 1$, $k=1,2$. We have from (3.4),

$$
\left(\frac{1}{2}|\xi|^{2}-|\eta|^{2}\right)^{2}+\sum_{i=1}^{q} Q_{i}^{2}(\xi)+\left|\sum_{i=1}^{q} \eta_{i} \nabla Q_{i}(\xi)\right|^{2}=\left(|\xi|^{2}+|\eta|^{2}\right)^{2} .
$$

Regarding the latter equality as an identity in $\mathbb{R}\left[\eta_{1}, \ldots, \eta_{q}\right]$, one finds

$$
\sum_{i=1}^{q} Q_{i}^{2}(\xi)=\frac{3}{4}|\xi|^{4}
$$

and

$$
\left\langle\nabla Q_{i}, \nabla Q_{j}\right\rangle=3 \delta_{i j}|\xi|^{2},
$$

where $\delta_{i j}$ is the Kronecker delta. Write $Q_{i}$ in the matrix form

$$
Q_{i}(\xi)=\frac{\sqrt{2}}{3} \xi^{\mathrm{t}} A_{i} \xi
$$

where $A_{i} \in \mathbb{R}^{p \times p}$ is symmetric. It follows then from (3.8) that the symmetric matrices $\left\{A_{i}\right\}_{1 \leq 1 \leq q}$ solve the Hurwitz matrix equations (2.1) (2.2) for $s=m=p$ and $r=q$; therefore

$$
q \leq \rho_{\text {symm }}(p) .
$$

If $q=1$, then (3.7)-(3.8) immediately yields $Q_{1}=\frac{\sqrt{2}}{3}|\xi|^{2}$ (the choice of sign of $Q_{1}$ is immaterial because we are free to change the sign of $x_{n}$ in (3.1)). Thus

$$
f(x)=x_{n}^{3}+\frac{3}{2} x_{n}\left(x_{1}^{2}+\ldots+x_{n-2}^{2}-2 x_{n-1}^{2}\right)+3 \sqrt{3} x_{n-1}\left(x_{1}^{2}+\ldots+x_{n-2}^{2}\right) .
$$


But the latter polynomial is exactly the solution (1.3) after a suitable rotation, namely,

$$
f\left(x_{1}, x_{2}, \ldots, x_{n-1}, x_{n}\right)=f_{0}\left(x_{1}, x_{2}, \ldots, \frac{\sqrt{3}}{2} x_{n-1}+\frac{1}{2} x_{n},-\frac{1}{2} x_{n}-\frac{\sqrt{3}}{2} x_{n}\right) .
$$

Finally, let us suppose that $q \geq 2$. Then (3.7) means that

$$
y(\zeta):=\frac{2}{\sqrt{3}}\left(Q_{1}(\zeta), \ldots, Q_{q}(\zeta)\right): S^{p-1} \rightarrow S^{q-1}
$$

is a quadratic map sending the unit sphere $|\zeta|=1$ to the unit sphere $|y|=1$. Note also that our assumption $q \geq 2$ implies by virtue of (3.8) that the image of $y\left(S^{p-1}\right)$ in $S^{q-1}$ is distinct from a point. Then one result of P. Yiu [6] provides an obstruction for a nonconstant quadratic map to exist if the dimension $q-1$ of the target sphere is too small. More specifically, let us denote by $\sigma(k), k \geq 1$, the minimal possible value of $l$ for which there exists a nonconstant homogeneous quadratic map $S^{k} \rightarrow S^{l}$. Then the theorem of P. Yiu [6, Theorem 4] (see also [7] for general polynomial maps) yields a recursive formula for $\sigma(k)$ :

$$
\sigma\left(2^{a}+b\right)= \begin{cases}2^{a}, & 0 \leq b<\rho\left(2^{a}\right) \\ 2^{a}+\sigma(b), & \rho\left(2^{a}\right) \leq b<2^{a} .\end{cases}
$$

We shall need only two easy consequences of the Yiu formula, namely, that $\sigma(m)$ is a nondecreasing function on $\mathbb{Z}^{+}$and

$$
\sigma\left(2^{a}\right)=2^{a}, \quad a \in \mathbb{Z}^{+} .
$$

In this set-up, one can rewrite the existence of a nonconstant quadratic map (3.11) as the lower estimate

$$
q-1 \geq \sigma(p-1) .
$$

Combining this with (3.10), we get after applying Proposition 2.1 that

$$
1+\sigma(p-1) \leq q \leq 1+\rho\left(\frac{p}{2}\right) .
$$

By our assumption $q \geq 2$; hence the right inequality in (3.13) implies that $\rho\left(\frac{p}{2}\right) \geq 1$, i.e. $p$ is even. We write this as $p=2^{\nu+1} p_{0}$, where $p_{0}$ is an odd number and $\nu \geq 0$. Then (3.13) and the definition of $\rho$ yield that

$$
1+\sigma\left(2^{\nu+1} p_{0}-1\right) \leq q \leq 1+\rho\left(2^{\nu}\right) .
$$

Notice first that $p_{0}=1$, because otherwise we would have $p_{0} \geq 3$ and by monotonicity of $\sigma$,

$$
\sigma\left(2^{\nu+1} p_{0}-1\right) \geq \sigma\left(3 \cdot 2^{\nu+1}-1\right) \geq \sigma\left(2^{\nu+2}\right)=2^{\nu+1} .
$$

But the latter contradicts the right inequality in (3.14) in view of $\rho\left(2^{\nu}\right) \leq 2^{\nu}$. Thus $p_{0}=1$ and we rewrite (3.14) as

$$
1+\sigma\left(2^{\nu+1}-1\right) \leq q \leq 1+\rho\left(2^{\nu}\right) .
$$

Now applying $\sigma\left(2^{\nu+1}-1\right) \geq \sigma\left(2^{\nu}\right)=2^{\nu}$ and $\rho\left(2^{\nu}\right) \leq 2^{\nu}$, we find

$$
1+2^{\nu} \leq q \leq 1+2^{\nu},
$$

where the right inequality is strong for $\nu \geq 4$. Thus the only possible values are $q=2^{\nu}+1, \nu=0,1,2,3$, and an easy check shows that all the values are compatible with (3.13). The corresponding values of $p, q$ and the resulting dimension $n$ are displayed in Table 1 . 
TABLE 1. Exceptional values of $p$ and $q$

\begin{tabular}{|c|c|c|c|}
\hline$\nu$ & $p=2^{\nu+1}$ & $q=2^{\nu}+1$ & $n=p+q+1$ \\
\hline 0 & 2 & 2 & 5 \\
\hline 1 & 4 & 3 & 8 \\
\hline 2 & 8 & 5 & 14 \\
\hline 3 & 16 & 9 & 26 \\
\hline
\end{tabular}

One can see that the last column contains exactly the dimensions of the isoparametric cubics found by É. Cartan that were mentioned in the Introduction. To finish the proof, it suffices only to show that for the values of $p$ and $q$ as in Table 1 , any solution $f$ of (1.1) is harmonic. But this is true because $q=2^{\nu}+1$ and $p=2^{\nu+1}$; hence from (3.5) we get $\Delta A(\bar{x})=p-2 q=-2$, and by virtue of (3.1) and (3.6), we find

$$
\Delta f=3(2+\Delta A(\bar{x})) x_{n}+3 \Delta B(\bar{x})=\sum_{i=1}^{q} \eta_{i} \Delta Q_{i}(\xi)
$$

On the other hand, for $q \geq 2$ the matrices $A_{i}$ in (3.9) by Proposition 2.1] are trace free, so that $\Delta Q_{i}=2$ trace $Q_{i}=0$. Thus, $\Delta f=0$ and we get (1.1)-(1.2). Applying Cartan's theorem finishes the proof.

\section{ACKNOWLEDGMENT}

The author would like to thank the referee for offering useful comments and suggestions.

\section{REFERENCES}

1. É. Cartan, Sur des familles remarquables d'hypersurfaces isoparamétriques dans les espaces sphériques, Math. Z. 45(1939), 335-367. MR0000169 (1:28f)

2. W. Y. Hsiang, Remarks on closed minimal submanifolds in the standard Riemannian $m$-sphere. J. Diff. Geom. 1(1967), 257-267. MR0225244 (37:838)

3. H. B. Lawson, Complete minimal surfaces in $S^{3}$, Ann. of Math. (2) 92(1970), 335-374. MR 0270280 (42:5170)

4. D. B. Shapiro, Compositions of quadratic forms, de Gruyter Expositions in Mathematics, 33. Walter de Gruyter \& Co., Berlin, 2000. MR1786291 (2002f:11046)

5. V. Tkachev, On a classification of minimal cubics in $\mathbb{R}^{n}$, in preparation.

6. P. Yiu, Quadratic forms between Euclidean spheres. Manuscripta Math. 83(1994), no. 2, 171181. MR1272181 (95b:55013)

7. R. Wood, Polynomial maps from spheres to spheres. Invent. Math. 5(1968), no. 2, 163-168. MR.0227999 (37:3583)

Department of Mathematics, Royal Institute of Technology, SE-10044 Stockholm, SWEDEN

E-mail address: tkatchev@kth.se 